\title{
EL DIBUJO INFANTIL COMO REPRESENTACIÓN DEL TIEMPO VIVIDO. MERLEAU-PONTY Y PIAGET EN LA COMPRENSIÓN DE LA TEMPORALIDAD*
}

\author{
CHILDREN'S DRAWING AS A REPRESENTATION OF LIVED TIME. MERLEAU- \\ PONTY AND PIAGET IN THE UNDERSTANDING OF TEMPORALITY
}

\author{
Jesica Estefanía Buffone ${ }^{* *}$ \\ CONICET - Universidad de Buenos Aires (UBA), \\ Buenos Aires - Argentina. \\ Université Lyon III, Jean Moulin, \\ Lyon - Francia.
}

Recibido agosto de 2018/Received August, 2018

Aceptado noviembre de 2018/Accepted November, 2018

\begin{abstract}
RESUMEN
En El dibujo infantil como representación del tiempo vivido. Merleau-Ponty y Piaget en la comprensión de la temporalidad, se analiza el desarrollo de la noción de temporalidad en la primera infancia desde una perspectiva fenomenológica, postulando al cuerpo y a la manipulación de los objetos en el mundo como factores cruciales en este proceso. En primer lugar, se desarrolla el análisis del dibujo infantil que realiza Merleau-Ponty en La prosa del mundo y en los cursos L'experiencie d'autrui, Structure et conflits de la conscience enfantine y Méthode en psychologie de l'enfant, presentes en los Cours de Sorbonne sobre infancia y pedagogía. En segundo lugar, se cotejan algunos trabajos de Piaget en torno a la temporalidad infantil, para ahondar en la tesis que Merleau-Ponty defendiera en los cursos sobre la expresión en torno al aprendizaje del tiempo en el mundo mismo. Por último, se analizará la crítica merleaupontyana a la concepción de Piaget de la representación del tiempo en los niños, para así elucidar la tesis del fenomenólogo francés al respecto y comprender desde allí la experiencia del tiempo de un sujeto en la vida adulta (o, más bien, la experiencia del tiempo sin más). De esta forma, la experiencia estética infantil es presentada por Merleau-Ponty como eminentemente fenomenológica, como la representación del tiempo vivido que aún no ha sido asimilada por la culturalización y las formas estandarizadas de vivir el tiempo y el espacio.
\end{abstract}

Palabras Clave: Temporalidad, Infancia, Cuerpo, Fenomenología.

\begin{abstract}
In Children's drawing as a representation of lived time. Merleau-Ponty and Piaget in the understanding of temporality, the development of the notion of temporality in early childhood is analyzed from a phenomenological perspective, postulating the body and the manipulation of objects in the world as crucial factors in this process. In the first place, the author develops the analysis of the children's drawing made by Merleau-Ponty in The Prose of the World and the courses L'experiece d'autrui, Structure et conflits de la conscience enfantine and Méthode en psychologie de l'enfant, all of them present in the Cours de Sorbonne about childhood and pedagogy. In the second place, some Piaget's works on infantile temporality are analyzed in order to delve into the thesis that Merleau-Ponty maintains in the courses on the expression around the learning of time in the world itself. Finally, the author analyzes and delves into the merleaupontyan critique of Piaget's conception of the infantile temporal experience, in order to elucidate the thesis of the French phenomenologist about it and understand from there the experience of subjective time in adult life (or, rather, the general experience of time). In this way, the infantile aesthetic experience is presented
\end{abstract}

* El presente artículo ha sido realizado en el marco del Proyecto de Investigación Científica y Tecnológica (PICT-Conicet) denominado La institución en debate: un acercamiento fenomenológico. Directora: Dra. Mariana Larison. Radicado en el Centro de Estudios Filosóficos «Eugenio Pucciarelli» (Academia Nacional de Ciencias de Buenos Aires). Periodo 2016-2019.

** Autor correspondiente / Correspondig author: jesicabuffone@ hotmail.com 
by Merleau-Ponty as eminently phenomenological, as the representation of lived time that has not yet been assimilated by the culturalization and by standardized ways of living time and space.

Key Words: Temporality, Childhood, Body, Phenomenology.

\section{Introducción}

Que la experiencia infantil ocupa en la obra de Maurice Merleau-Ponty un lugar privilegiado, es, desde una lectura no demasiado profunda, un rasgo que salta a primera vista. Las razones que sustentan dicho privilegio no son, sin embargo, tan evidentes. Incluso, no es siquiera evidente qué es aquello que, dentro de la experiencia infantil, goza para el fenomenólogo francés de una superioridad por sobre la experiencia de los adultos. En este trabajo, se ensayará una respuesta a esta pregunta que, en el marco de la filosofía merleaupontyana, no puede ser respondida sin apelar a la pintura o, incluso, a la poesía. Tal vez desde este punto podemos empezar a acercarnos a una posible respuesta en torno a la valoración de la experiencia infantil: esta no puede ser definida sin apelar a lo poético, a lo lírico, a un registro que nos acerca a aquello que no se nos muestra como evidente, al contacto con una experiencia que nos habla, en definitiva, de la experiencia misma.

La representación infantil del tiempo vivido expresada en el dibujo, será una de las principales entradas para comprender, desde la obra de Merleau-Ponty, la particularidad y la relevancia de la experiencia de los niños. En el dibujo infantil, se ve el entrelazo de la dimensión temporal con la espacial, que es, en definitiva, nuestra única manera de aproximarnos a una comprensión del tiempo vivido. Es por ello que, en este trabajo, se analizará la representación de la experiencia por parte de los niños en el dibujo, para comprender desde allí nuestra vivencia de la temporalidad. La afirmación que Merleau-Ponty expresa en Le monde sensible et le monde de l'expression (2011) según la cual, tal como sostenía Piaget, el tiempo se aprende en el mundo, será central para establecer la ligazón entre la representación del espacio y la vivencia del tiempo. Para ahondar en dicho interrogante, se desarrollará, en primer lugar, el análisis del dibujo infantil que realiza Merleau-Ponty en $\mathrm{La}$ prosa del mundo y en los cursos L'experiencie d'autrui, Structure et conflits de la conscience enfantine y Méthode en psychologie de l'enfant, presentes en los Cours de Sorbonne sobre infancia y pedagogía (Merleau-Ponty, 2001). En segundo lugar, se explorarán algunos trabajos de Piaget en torno a la temporalidad infantil, para ahondar en la tesis que Merleau-Ponty defendiera en los cursos sobre la expresión. Por último, se analizará la crítica merleaupontyana a la concepción de Piaget de la representación del tiempo en los niños, para así elucidar la tesis del fenomenólogo francés al respecto y comprender desde allí la experiencia del tiempo de un sujeto en la vida adulta (o, más bien, la experiencia del tiempo sin más). De esta forma, la manera en la que el niño representa el mundo que lo rodea como una dimensión en donde tiempo y espacio se conciben como indiscernibles, nos acercará a una mejor comprensión de aquel rasgo privilegiado de la experiencia infantil que nos conecta nuevamente con la vivencia misma de las cosas, la cual ha sido sepultada por el aprendizaje y la cristalización de formas establecidas de ordenar y percibir nuestro entorno.

\section{2. ¿Qué nos muestra el niño en la experiencia estética? El tiempo como un río que fluye con nosotros}

La experiencia estética en el marco de la filosofía de Maurice Merleau-Ponty ha recibido gran atención por parte de muchos especialistas (Álvarez Falcón, 2008; Battán Horenstein, 2010; Carbone, 2011). Sin embargo, el rol de la infancia en el marco de su filosofía y el diálogo que el filósofo francés sostuvo con la psicología del desarrollo, han sido temas raramente abordados por quienes se dedican al estudio de su obra, razón por la cual son escasos los antecedentes en torno a estas problemáticas (Bimbenet, 2002; Saint Aubert, 2006). De esta forma, se hace imperioso un análisis de esta dimensión de la filosofía merleaupontyana, para esclarecer así un intercambio tan rico y complejo como aquel que ha tenido lugar entre la fenomenología y la psicología del desarrollo.

En Le monde sensible et le monde de l'expression. Cours au Collège de France. Notes, de 1953 (2011), Merleau-Ponty sostiene que el 
sentido del tiempo nos es dado como una configuración cultural, a partir de lo cual la percepción es definida por Merleau-Ponty como " "una máquina de vivir' el tiempo" (Merleau-Ponty 2011, p. 190). "Mi cuerpo no es solamente un aparato de realizar los anclajes en el espacio", sino que también realizará "los anclajes en el tiempo" (Merleau-Ponty, 2011, p. 190). El tiempo, en definitiva, será lo que organizará en alguna medida el campo (champ) perceptivo. Por esa razón, Merleau-Ponty sostiene que

la relación entre mí mismo y los símbolos temporales no puede ser una relación de sentido centrífuga, porque el yo que le da sentido a los símbolos sociales está él mismo inserto por la percepción en un campo en el cual la temporalidad institucional forma parte. (Merleau-Ponty, 2011, p. 190).

Por esa razón, Merleau-Ponty tomará la afirmación de Jean Piaget según la cual "los niños aprenden el tiempo en el mundo", lo cual parece ser para el fenomenólogo la única forma, en definitiva, de poder acceder a él.

El niño, con su construcción aún fluctuante de su propio pasado, da cuenta del carácter convencional y cultural de "las máquinas de vivir el tiempo". Sin embargo, ¿en qué consiste la vivencia del tiempo en los niños? ¿Cuál es el aspecto relevante de la experiencia infantil sobre la temporalidad? Para poder dar respuesta a estas preguntas, debemos, en primer lugar, aproximarnos a los análisis merleaupontyanos del dibujo infantil, en donde la representación espacial es indiscernible de la matriz temporal que la contiene y le da sentido.

\subsection{El arte como reconfiguración de lo percibido}

$\mathrm{El}$ arte ha venido a salvarnos. Nos acerca a la experiencia, nos la devuelve y, ante todo, nos ubica en un medio afectivo en donde, irremediablemente, nos situamos en medio de las cosas. La obra de arte disuelve la lejanía impuesta por un cientificismo que nos ha hecho olvidar el mundo que nos rodea, reconfigurando en el acto mismo de expresión la experiencia que subtiende y da sentido a lo expresado. Por esa razón, MerleauPonty apelará a la historia del arte para dar cuenta del cambio en la forma de representar el espacio circundante y, al mismo tiempo, analizar desde allí la manera en la que los sujetos de una determinada época se relacionan con su entorno.

En El mundo de la percepción. Siete conferencias (2002), el arte será para Merleau-Ponty una metáfora de aquello que la fenomenología querrá rescatar desde una mirada filosófica: el mundo de la vida, la experiencia encarnada, la afectividad de las cosas que se nos manifiestan. En el marco de la teoría merleaupontyana, el arte es entendido como una manifestación que produce un corrimiento de la mirada que de forma habitual arrojamos sobre el mundo y nos revela (poniendo de manifiesto la manera en que el mundo sale a nuestro encuentro) nuestro lugar en él. Para Merleau-Ponty, el arte no es imitación, ni nace del gusto estético del artista, sino que es un proceso de expresión, en el cual el mismo recaptura y convierte en objetos visibles lo que sin él, se mantendría callado en la vida separada de cada conciencia. El artista capta "la vibración" de las cosas, el halo que las recubre y que implica su existencia misma. La presencia de este sentido incipiente, oculto y disperso detrás de todas las cosas nos condena a vivir, como afirma Merleau-Ponty, en medio del sentido (MerleauPonty, 1984).

La obra de arte subvertirá los parámetros del tiempo histórico-objetivo, producirá una ruptura en la continuidad temporal; "su ser será un puro aparecer" (Álvarez Falcón, 2008) que, produciendo una herida en el tiempo, desbordará las estructuras subjetivas destruyendo los horizontes de los objetos. De esta manera, el modo en que los objetos se nos aparecen en la obra de arte rompe, tal como sostiene Heidegger (1996), con las relaciones habituales que entablamos con el mundo. La pintura de Cézanne suspende para el filósofo "estas costumbres y revela el fondo de naturaleza inhumana en el cual se instala el hombre" (Merleau-Ponty, 1977, p. 250). La obra de Cézanne descubre la relación que guardamos con nuestro entorno revelando el tiempo que habita entre los distintos escorzos del objeto. El arte, entonces, rompe con "la continuidad del presente del tiempo vivido" (Álvarez Falcón, 2008, p. 92) poniendo al descubierto el transcurrir del tiempo mismo. De esta forma, Cézanne ha puesto al descubierto que nuestra relación con el espacio no es la "de un puro sujeto descarnado", sino la de "un habitante del espacio con su medio familiar" (MerleauPonty, 2002, p. 23). El mundo nos interpela como seres eminentemente corporales suscitándonos 
determinadas conductas y reacciones, debido a que las cosas "no son objetos neutros", sino que poseen un "halo" (Merleau-Ponty, 2002, pp. 30-31) que le habla a nuestro cuerpo y frente al cual no podemos permanecer inmutables.

De una manera análoga a la del artista, el historiador recuperará aquel "sentido naciente", aquel sentido disperso presente en los hechos, el cual será expresado en su relato sobre los acontecimientos. De esta manera, el sentido y las significaciones se desprenden de todo aquello que hacemos o producimos en el mundo; no hay nada que carezca de una significación. "Estamos condenados al sentido" (Merleau-Ponty, 1984, p. 19) ya que todas nuestras acciones adquirirán un significado en la historia, y será el historiador, entre otros, quien "reanude" el sentido que en forma incipiente se esboza en el mundo de la vida. Es allí donde los artistas, los historiadores, los filósofos y los niños entran en escena, para barrer de nuestra experiencia aquello que se desprende casi como un daño colateral, como una producción marginal pero inherente. El artista, el filósofo, el historiador y el niño se asemejan en su voluntad (en unos expresa, en otros fortuita) de sustraer el sentido de los acontecimientos, poniendo en un primer plano las significaciones que le dan unidad. Sin embargo, ¿en qué medida el dibujo de los niños puede ser analizado en el mismo nivel que la pintura de Cézanne? Quizás lo que en este último es resultado de la pericia pictórica, en los niños no es más que el reflejo de una existencia que aún no ha sido incorporada a los aparatos culturales que mesuran y ordenan nuestra experiencia (formas de medir el tiempo, de representar la realidad, de analizarla, de volverla un todo universalmente accesible). En este sentido y teniendo en cuenta aquello que el fenomenólogo francés rescata de la pintura de Cézanne, la representación que del mundo realizan los niños puede ser cotejada como otra vía que nos enfrenta con nuestras habitualidades representacionales. El niño recupera esa experiencia que para Merleau-Ponty la ciencia ha sepultado y que la pintura moderna (encarnada en Cézanne y Manet) se encargó de rescatar. A continuación, se desarrollarán las particularidades de este proceso.

\subsection{El dibujo infantil como síntesis de la experiencia}

En el curso Méthode en psychologie de l'enfant (2001), la pintura italiana (como paradigma de la pintura clásica y refiriendo con esta denominación al renacimiento italiano), se ubica en las antípodas del impresionismo, corriente que será puesta en diálogo con el dibujo de los niños. En el caso de la pintura italiana, el objeto pintado por el artista guarda una relación de absoluta correspondencia con el modelo; el artista intenta imitar de alguna manera aquello que tiene ante sus ojos y la virtud del pintor radicará en el nivel de fidelidad que posee a la hora de capturar la cosa vista. La pintura, en este caso "rivaliza con la naturaleza" y su objetivo será reemplazarla. De esta forma, la pintura no es algo "personal", sino que puede ser realizada de forma similar por otros artistas, en la medida en que su virtuosismo yace en la pericia respecto al manejo de técnicas. En la pintura clásica, la subjetividad del artista desaparece no solo frente al intento de capturar de la manera más fiel posible el espectáculo de la naturaleza, sino también frente al despliegue estandarizado de las técnicas pictóricas. Con los expresionistas y, en particular, con Manet, aparece otra idea de la pintura, ya no asociada con un registro fiel de la realidad, sino más bien a la expresión, esto es, a la aparición de la subjetividad del artista en medio de lo representado. La pintura ya no es más el mundo mismo, sino más bien una forma de representarlo; ya no se trata de reproducir el objeto, de aprehender los detalles, sino de capturar el movimiento interno de la cosa y hacerlo evidente. La figura del artista pasa a un primer plano y su presencia acompaña, en igual medida, la representación misma de lo pintado: pintar es, antes que una representación exacta de lo real, "una firma" (Merleau-Ponty, 2001, p. 518) que da cuenta de una manera determinada de ver y aproximarse al mundo. El pintor dibuja su contacto con la cosa misma, antes que la cosa en sí misma, intentando dar cuenta de la relación que poseemos con el mundo que nos rodea.

De la misma manera, los niños nos ponen con sus dibujos frente a la relación que poseemos con las cosas. Sin embargo, en el dibujo infantil la subjetividad de la obra y la posible objetividad de la misma, se traban en una relación recíproca. Por un lado, el niño intenta ponernos frente al contacto que él mismo establece con las cosas y, por otro, hay también un intento por darnos "la presencia real" de la cosa (Merleau-Ponty, 2001, p. 519). Si bien, según Merleau-Ponty, podemos decir que el dibujo del adulto es más "objetivo" 
que el del niño, el mismo no nos aporta "la realidad de la cosa", ya que el dibujante adulto "no nos da la situación vivida con la cosa". El dibujo adulto no es sino el recorte de una perspectiva de la cosa entre tantas otras perspectivas posibles y no es, tal como sostiene respecto a la pintura de Cézanne, "la cosa total en su simultaneidad imperiosa" (Merleau-Ponty, 2001, p. 519). El dibujo adulto no es una representación del mundo tal cual lo vemos, sino meramente una forma específica de expresión, siendo el dibujo infantil otra manera alternativa de expresarse. Debemos, entonces, dejar de pensar al dibujo como una construcción en términos de "equivalencia" o "proyección" de la realidad y reconsiderarlo como la expresión de nuestra comunicación con las cosas.

El dibujo infantil es "una relación total y global con el objeto", en la medida en que el niño no expresa solamente el aspecto visual del mismo, sino que trata de atrapar su experiencia particular con las cosas, su "impresión" con respecto a ellas. En el dibujo, el niño no se centra en el aspecto visual, sino que intenta rescatar la experiencia que ha tenido lugar cuando tuvo contacto con ese objeto. El niño no intentará para Merleau-Ponty reproducir el aspecto visible de las cosas, sino que más bien evocará situaciones (construidas por objetos, personajes, colores, etc.) que recuerden esa experiencia. Habría, de esta forma, una aprehensión global de la experiencia, que el niño intentará reconstruir apelando a elementos clave de ésta y no a una reconstrucción término a término de los elementos visuales que la constituyen: el niño capta en su reconstrucción de la experiencia, su relación con los otros. A diferencia del adulto, el niño no establece con el mundo una relación entre él como contemplador y el mundo como objeto contemplado. $\mathrm{Al}$ respecto, Merleau-Ponty sostiene que "la sensorialidad está subtendida por una relación con el mundo que no es una relación sensorial, sino una relación total y afectiva con las cosas" (Merleau-Ponty, 2001, p. 521). Toda cualidad "es reveladora de un cierto modo de sincronización del sujeto encarnado con el mundo". Sin embargo y en virtud de esta sincronía entre el sujeto y su entorno, la pregunta por la posibilidad de pensar en rasgos permanentes en el dibujo infantil sale a nuestro encuentro: ¿hay rasgos o constantes en los dibujos que realizan los niños independientemente de la cultura que los acoge? En Structure et conflits de la conscience enfantine (2001), Merleau-Ponty desestima esta cuestión por considerarla en verdad un "falso problema" (Merleau-Ponty, 2001, p. 210). A la luz de las influencias marxistas que han nutrido su teoría (Wallon [1965] en particular y sobre todo respecto a este tema), para el fenomenólogo francés "es imposible separar aquello que viene de la cultura y aquello que pertenece propiamente al niño" (Merleau-Ponty, 2001, p. 210). Los productos culturales que el niño encuentra en el medio en el que vive (la publicidad, las vidrieras, el mobiliario) influyen desde un primer momento en aquello que representa.

De esta forma, el dibujo infantil debe ser comprendido como expresión, en donde se lleva a un solo plano aquello que percibimos en profundidad. Es así como la proyección en los dibujos del niño no debe ser comprendida como una imitación de aquello que ve, como una traducción directa de aquello que percibe, sino como una expresión de su experiencia. Aquello que Merleau-Ponty denomina perspectiva geométrica (la cual refiere al acto de "traducir" sistemáticamente y según leyes precisas lo percibido) ha sido considerada como reproducción fiel de nuestra visión. Sin embargo, ello sucede porque una larga tradición artística ha acreditado esta equivalencia entre el mundo percibido y el dibujo, ocultando de esta forma el carácter expresivo de esta unión. Cuando un niño utiliza otro procedimiento como la proyección (por ejemplo, cuando representa un cubo a partir de cinco cuadrados yuxtapuestos) el mismo es tan válido como el dibujo en perspectiva, ya que el niño no hace sino expresar la forma en la que percibe el mundo.

Merleau-Ponty desarrollará su interpretación del dibujo infantil a partir, en primer lugar, de la obra Le dessin enfantin de Georges-Henri Luquet (Piaget, 1978, cap. 3). En primer lugar, para Luquet, el dibujo es considerado por el niño como un juego, que distinguirá claramente de los croquis explicativos que elabora para "mostrar algo". El dibujo posee asimismo el carácter de una "obra", de una "performance personal" (Merleau-Ponty, 2001, p. 212), en donde habría un despliegue de producción a partir del cual se producen muchos dibujos en muy poco tiempo. Para este autor, en la medida en que hay una ligazón muy fuerte entre el dibujo y las cosas del mundo, habría cierto "realismo" pictórico al intentar representar las cosas tal cual son. El segundo rasgo que distingue Luquet del dibujo infantil es la plasticidad. Si los 
niños tienen que corregir algo, no borran todo, sino que corrigen "mentalmente". El elemento visual no es el "estimulante esencial" en el dibujo del niño. Asimismo, el sentido del dibujo tiene una significación "retrospectiva", en la medida en que puede otorgarle una significación diferente a la inicial que le parece más apropiada. La significación del dibujo se impone como necesaria en virtud del estatus del mismo, el cual tiene una realidad análoga a la de los objetos: en virtud de su existencia, necesariamente debe significar algo. El realismo del dibujo hace que el niño incluso llegue a olvidarse que es su creación. Puede enojarse con él o tratarlo con afecto. En tercer lugar, hay cierta impermeabilidad respecto a la experiencia. El acto de dibujar es un "ritual" en donde el objetivo no es reproducir lo que se ve. En cuarto lugar, Luquet nota que habría un modelo interno que guía el dibujo. No representa lo que ve, sino lo que sabe que son los elementos constitutivos de lo que ve. Luquet lo denomina realismo intelectual, en la medida en que se efectúa una reconstitución del objeto, en donde se muestra lo que de otra manera no veríamos. Así un niño dibujará una casa, pero dejará ver al mismo tiempo algunas partes de su interior. De esta forma, el dibujo infantil será para Luquet eminentemente realista ${ }^{1}$ en virtud de su continua referencialidad a los objetos. De esta forma, Luquet considera que hay una simetría o correspondencia entre lo que percibimos y el mundo ("postulado de constancia"), por lo que la fotografía es considerada como la expresión más fiel de nuestro entorno.

Para Merleau-Ponty, el estudio del dibujo infantil por parte de Luquet desde la perspectiva del realismo visual (como una etapa eminentemente ideal), hace que este último sea considerado como una expresión degradada del dibujo del adulto. Por ejemplo, el estadio de realismo fallido es caracterizado negativamente a partir de la torpeza, casualidad y falta de atención propios de esta etapa. La descripción negativa de Luquet se funda para Merleau-Ponty en la creencia según la cual la percepción corresponde de derecho rigurosamente al mundo exterior del adulto y que por eso el niño tendría que percibir de la misma manera que nosotros. Para el fenomenólogo, en cambio, "el niño trata de representar toda la realidad existente, mientras que nuestro modo de síntesis suprime todo aquello que no se pueda ver desde un solo punto de vista" (Merleau-Ponty, 2001, p.
216). De esta manera, tanto adultos como niños sacrificarían algo en el dibujo: lo adultos lo hacen por la apariencia visual y los niños, para expresar el objeto en su totalidad.

Estamos inmersos, sostiene Merleau-Ponty, en la "ilusión objetivista" según la cual "el acto de expresar, en su forma normal o fundamental, consiste, dada una significación, en construir un sistema de signos tal que a cada elemento del significado corresponde un elemento del significante, es decir, a representar" (Merleau-Ponty, 1971, p. 205). La perspectiva planimétrica ${ }^{2}$ intenta ofrecer "una notación del mundo que sea válida para todos", a partir de la cual se "fija la perspectiva vivida" y, en tanto que construye una imagen que puede ser traducida como cualquier otro punto de vista, "es la imagen de un mundo en sî" (Merleau-Ponty, 1971, p. 207). En la medida en que la "deformación" que este tipo de perspectiva produce es "sistemática" y se aplica a todos los elementos del dibujo, produce la ilusión de ver las cosas desde "el conocimiento que puede tener de una visión humana un Dios que no se sumerge en la finitud" (Merleau-Ponty, 1971, p. 207). El dibujo del niño, en cambio, expresa otra forma de aproximarnos a nuestro entorno, no ya desde esta pretensión de asir un punto de vista universal, sino "nuestra relación con el mundo". El dibujo infantil no intenta reconstruir para Merleau-Ponty un punto de vista objetivo sobre el mundo, sino que señala nuestro contacto mismo con las cosas, en tanto ellas nos invocan de alguna manera. La expresión infantil nos propone una forma de expresión "elíptica" según la cual, cuando se representa un espectáculo determinado, se invocan todos los elementos que intervienen en él, ubicándolos en un mismo plano de coexistencia. "Todos los elementos del espectáculo son señalados sin equívoco y sin superposición" (Merleau-Ponty, 1971, p. 206). En el dibujo infantil se revela la presencia de nuestro cuerpo en el mundo, "el sello de nuestra finitud" y "la substancia secreta del objeto". Así, MerleauPonty explica:

La perspectiva planimétrica nos da la finitud de nuestra percepción, proyectada, aplanada, convertida en prosa bajo la mirada de un dios, los medios de expresión del niño, cuando ellos hayan sido retomados deliberadamente por un artista en un verdadero gesto de creador, nos darán por el contrario la resonancia secreta por la cual nuestra finitud se abre al ser del 
mundo y se hace poesía. (Merleau-Ponty, 1971, p. 209).

En los dibujos infantiles, los niños ponen todos los elementos de un relato en un mismo plano. Es decir, en una sola imagen son convocados todos los momentos, acciones y personajes que forman parte de una historia. Los niños conjugan el devenir de una historia en un solo plano, que los conecta y que hace visibles las relaciones entre sus partes. Lejos 'del adulto 'razonable', que piensa el tiempo como una serie de puntos temporales yuxtapuestos" (Merleau-Ponty, 1971, p. 216), el niño nos pone frente al plexo temporal mismo, frente a la dinámica elíptica del tiempo vivido en el que el presente nos remite como un haz al pasado $\mathrm{y}$ al porvenir. Es por ello que Luquet se pregunta si esto expresa una temporalidad diferente a aquella de los adultos. "Para el niño hay un encastre del pasado en el presente mientras que el adulto se representa el tiempo como un desarrollo de instantes netamente separados que se suceden" (MerleauPonty, 1971, p. 217). Esta concepción del adulto es filosóficamente "insostenible": es imposible pensar en el presente sin una implicación con el pasado y con el futuro, lo cual es expresado de alguna manera en la narración gráfica del niño. Es así como el mundo expresivo del niño nos ofrece otra dimensión del acto mismo de expresión, en donde la dimensión estética se cruza, al igual que en el artista, con la dimensión temporal. Esto lleva a pensar el dibujo infantil como implicando "un modo de comunicación diferente al nuestro, y que es sobre todo afectivo" (Merleau-Ponty, 1971, p. 217). El niño trata de reproducir la cosa en sí y en este sentido, el dibujo infantil es más subjetivo que el del adulto en la medida en que "se libera de la apariencia" pero a su vez es más realista porque intenta reproducir la cosa tal cual es. Por lo tanto, el dibujo del niño es una "expresión del mundo y jamás una simple imitación" (MerleauPonty, 1971, p. 217), tomando en un sentido pleno la conjunción entre aquel que percibe y la cosa percibida; "significa, expresa el mundo".

Esta forma de expresión propia de los niños refleja para Merleau-Ponty la naturaleza misma del acto expresivo y, como contrapartida, la operación que se efectúa en el acto mismo de la percepción. Las cosas que percibimos suscitan en nosotros determinadas emociones, determinadas imágenes que hacen imposible restringir la percepción a una mera aprehensión del mundo en términos de equivalencia. Tal como decía Goethe, "no hay color que no suscite una actitud". Cada cualidad sensible suscita y es "símbolo de una manera de ser" (Merleau-Ponty, 1971, p. 219). En el dibujo del niño, al igual que en la pintura de Cézanne, se transmite una "significación total" en la que todos los sentidos se van encadenando. El dibujo de los niños nos muestra que los cosas no pueden ser concebidas como objetos neutros, como compuestos de cualidades que se van sumando una a una hasta constituir el objeto total. Las cosas desprenden "una atmósfera moral" (Merleau-Ponty, 2002, p. 28) suscitando alegría, tristeza, angustia, etc., e invocan la totalidad de nuestro cuerpo:

\begin{abstract}
decir que la miel es viscosa y decir que es azucarada son dos maneras de decir lo mismo, o sea, cierta relación de la cosa con nosotros, o cierta conducta que nos sugiere o nos impone, cierta manera que tiene de seducir, de atraer, de fascinar al sujeto libre que se ve enfrentado con ella. La miel es cierto comportamiento del mundo para con mi cuerpo y para conmigo. (Merleau-Ponty, 2002, p. 29).
\end{abstract}

De esta forma, afirma Merleau-Ponty, las cualidades no están yuxtapuestas unas con otras, sino que serían "idénticas" en la medida en que expresan una misma forma de ser, por lo que la unidad de la cosa no es algo que esté por detrás de las cualidades, sino que es reafirmada por cada una de ellas. De esta forma, las cosas tienen lo que Cézanne llamaba un "halo", que era aquello que los artistas debían rescatar. Los niños, en su intento de reflejar el mundo que los rodea, han tenido éxito en esa tarea.

\section{La temporalidad infantil en la psicología genética}

El análisis del dibujo infantil como representación de una situación determinada, de un mundo específico que le habla al niño, es inherente a una vivencia determinada del devenir temporal. ¿Se origina acaso en el contacto con una temporalidad absoluta, pasible de ser asida por todos y cada uno de nosotros, y que espera a ser develada por un sujeto que puja por alcanzar su madurez? La construcción simultánea y no episódica que se evidencia en el dibujo del niño, nos lleva a preguntarnos por la génesis misma de nuestra noción 
de tiempo, y por la ligazón entre la representación del espacio y la matriz temporal que la subtiende. En Le monde sensible et le monde de l'expression (2011), Merleau-Ponty tomará la afirmación de Jean Piaget según la cual "los niños aprenden el tiempo en el mundo". Sin embargo, ¿de qué habla Piaget cuando sostiene que el tiempo se aprende en el mundo?

Para Piaget, no hay un tiempo interno que se proyecta hacia el exterior, sino que la organización temporal se da por la acción del niño en el mundo. El niño, debido a su egocentrismo intelectual ${ }^{3}$, considera al tiempo físico como "duraciones internas contraíbles y dilatables en función de los contenidos de la acción", por lo que "después no llega a la idea de un tiempo homogéneo" (Piaget, 1992, p. 209). ¿Dónde buscar entonces la fuente del tiempo? ¿En el interior del sujeto? ¿La "duración vivida" es el tiempo verdadero? En primer lugar, Piaget sostiene que el tiempo físico del niño empieza por ser egocéntrico en la medida en que no hay una diferenciación clara con el entorno. Asimismo, ignora las relaciones objetivas y produce una deformación de las cosas, pero el tiempo aún no es atribuido inductivamente a las mismas:

Así como el niño presta a los objetos un conjunto de cualidades sacadas de la acción propia (animismo, artificialismo, finalismo, etc.) así también, o para decirlo mejor por el hecho mismo, materializa su yo y no concibe su propia actividad más que en función de esos datos físicos y espaciales que no sabe disociar de ella. (Piaget, 1992, pp. 210-211).

El tiempo primitivo del niño resulta, entonces, de una indiferenciación del tiempo del sujeto y de los objetos. La duración se va a construir por referencia a estos últimos, por lo que tiempo físico y tiempo psicológico se apoyan mutuamente. La duración interior que experimenta el niño es el tiempo de la acción propia, es decir, la relación entre el sujeto y los objetos sobre los que actúa. Durante el período sensorio-motor, los objetos perseguidos y la actividad misma "constituyen una sola totalidad de sucesión y de duración" (Piaget, 1992, p. 211), habiendo un solapamiento entre la acción y el objetivo perseguido. Cuando se objetiva el tiempo físico, hay subjetivación del tiempo psicológico, ya que "la acción sólo es creadora bajo condición de unirse a los objetos". De esta forma, los niños más pequeños (para quienes la edad, incluso, es la estatura) construyen la temporalidad desde una perspectiva completamente egocéntrica. Por esa razón, Piaget se pregunta si acaso el niño "falla" en su representación del tiempo antes del tercer estadio. Antes de la consolidación de las operaciones formales, el niño no ubica los eventos en serie de forma coherente y no calcula las duraciones. Las sucesiones se ven alteradas y los eventos son narrados con un orden alternativo al que efectivamente sucedieron. Por otra parte, el tiempo psicológico es el tiempo de la acción en curso, siendo el interés del niño por la actividad realizada un dinamizador de la misma (aquellas actividades que resultan tediosas para el niño producirán un efecto por el cual el tiempo parece transcurrir más lentamente. El disfrute, en cambio, provocará el efecto opuesto). Por ello, Piaget sostiene:

\begin{abstract}
Cuando la acción en curso es un trabajo y no una contemplación pasiva, los niños no evalúan el tiempo de la acción propiamente dicha en términos de estados de conciencia (es decir, por introspección directa), sino en términos objetivos o, mejor dicho, "realistas", fundándose en los resultados de la acción o en su velocidad. (Piaget, 1992, p. 244).
\end{abstract}

La toma de conciencia ${ }^{4}$ del devenir temporal, irá para Piaget "de la periferia al centro" en la medida en que el resultado de la acción será central para estimar la duración de la misma. De esta manera, afirma, "el tiempo interior no es la forma productiva del tiempo", sino que está ligada a nuestro accionar sobre las cosas. Sin embargo, las duraciones y las sucesiones (estas últimas expresadas, por ejemplo, en la utilización de algún elemento como una vara para alcanzar otra cosa), no expresan la posesión de "un esquema de tiempo homogéneo", sino que son meramente coordinaciones de acciones particulares. Es así como Piaget sostiene que

nos vemos llevados a suponer que la forma original del tiempo es un esquema que depende a la vez de la organización del universo físico y de la acción propia; por lo tanto, el tiempo es egocéntrico, lo que de ningún modo significa egocéntrico en estado puro. (Piaget, 1992, p. 262). 
Dicho esquema ya se puede advertir para Piaget incluso durante los primeros meses de vida: el llanto del bebé cuando la espera se hace larga evidencia cierta vivencia prematura del tiempo, llegando a la conclusión de que el esquema práctico del tiempo se elabora desde el estadio de la inteligencia sensorio-motora.

Respecto a la narración de eventos, antes de los 7 u 8 años los niños ordenan los eventos siguiendo un hilo completamente egocéntrico, esto es, no hay una concatenación temporal, sino que la misma se organiza egocéntricamente. Entre los 2 y los 4 años, cuando los niños intentan narrar un evento de interés, amontonarán una serie de eventos yuxtapuestos, ordenados en pequeñas series o parejas. La pregunta que se hace Piaget al respecto es si en definitiva esta yuxtaposición de la narración traduce un desorden interno o simplemente es un defecto de la exposición. Al respecto, concluye que estas dos incoherencias (esto es, el desorden narrativo y el desorden interno propio de la memoria) no hacen sino reflejar un mismo y único problema. Hay una coordinación del comportamiento social y del comportamiento intelectual, en la medida en que cuando el niño aprende a narrarle un evento a los otros, podrá narrárselo a sí mismo, organizando de esta forma su memoria. A partir de una experiencia de secuenciación de eventos ${ }^{5}$, Piaget advierte que, luego de que los niños que habían efectuado la secuenciación incorrectamente eran advertidos de este hecho y se les presentaba la secuencia correcta (aceptando la nueva versión de la misma), muchos incurrían nuevamente en el mismo error, repitiendo la secuencia malograda de la primera experiencia con las imágenes ${ }^{6}$. $\mathrm{Al}$ respecto, concluye:

Esta dificultad para reconstruir otro relato, por tanto, a volver atrás para seguir una nueva dirección, o, de manera general, para considerar un relato como una hipótesis que, según la necesidad, se puede anular para concebir otras, resulta directamente de esta irreversibilidad del pensamiento de los pequeños, dirigido siempre en el sentido de la acción a la manera de una pura experiencia mental, sin regreso ni movilidad operativa. (Piaget, 1992, p. 266).

De esta manera, Piaget muestra cómo la sucesión se consigue con la reversibilidad del pensamiento, porque narrar una historia de forma concatenada exige tener la estructura de "anterior a $x$ ", "posterior a $y$ ". Recién a los 7 u 8 años, los niños advierten estas relaciones y la complejidad de su reconstrucción radica en el enmarañado entramado de las vivencias de un sujeto: un relato escolar se mezcla con vivencias propias de la vida familiar, las relaciones con los pares, etc. La reconstrucción de una serie de eventos implica la organización y la diferenciación de la misma de otras series que interfieren. De esta manera, "reconstruir el pasado será, pues, reconstruir esos diversos desenvolvimientos, pero con sus cadencias propias" (Piaget, 1992, p. 266).

Para Piaget, tanto el tiempo físico como el tiempo propio de las acciones psicológicas consiste en una "coordinación de movimientos y velocidades" (Piaget, 1992, p. 267), por lo cual es sumamente difícil la reconstrucción del orden de los eventos cuando aún no se ha conseguido la reversibilidad operativa. En este proceso, hay un paso del egocentrismo irreversible al "agrupamiento operatorio o coordinación objetiva y reversible" (Piaget, 1992, p. 269), en donde el yo comienza a ser un elemento más en medio de esa organización. Es por ello que en el período sensorio-motor, el tiempo se confunde con el espacio o la materialidad de los movimientos, ya que, en la medida en que

la velocidad no ha sido adquirida bajo una forma operativa, es decir, como una relación entre el espacio recorrido (o el trabajo realizado, etc.) y esta dimensión común a las distintas velocidades, que es precisamente el tiempo, el orden temporal se confunde con el orden espacial y la duración con el camino recorrido. (Piaget, 1992, p. 271).

Por esa razón, los niños juzgarán la duración de una acción determinada por el resultado obtenido o el trabajo realizado para llevarla a cabo (de hecho, "más rápido" significa para un niño "más tiempo"). El "tiempo práctico" se especializa en relación con la acción realizada y hay tantas series temporales como acciones realizadas, sin que haya aún un tiempo único que vincule unas con otras. Luego, las nociones de sucesión y de duración serán construidas a partir de los esquemas consolidados en el período sensorio motor. De esta forma, el niño "podrá decir que estaba en $\mathrm{B}$ después de haber estado en $\mathrm{A}$, y en $\mathrm{C}$ después de haber estado en B”, pero aún la sucesión y la 
distancia temporal se confunde con la espacial. El tiempo correspondiente a este estadio es "un tiempo sin velocidades", por lo que el antes y el después guardan entre sí solamente una relación espacial. Por ello, Piaget concluye que "la construcción del tiempo comienza cuando las distintas velocidades se comparan entre sí, velocidades de las actividades humanas tanto como de los movimientos materiales" (Piaget, 1992, p. 271). Piaget menciona la siguiente situación: "cuando un móvil pasa a otro, o partiendo de un mismo punto y yendo a la misma dirección, llega más lejos que el otro, todos los pequeños concuerdan en decir que va más de prisa" pero si el pasar es invisible (por ejemplo, la carrera se desarrolla dentro de túneles) "el cálculo de las velocidades es entonces inexacto" (Piaget, 1992, p. 275). La velocidad no es aún una noción que se construye como una relación entre el tiempo y el espacio recorrido. Antes de esta coordinación, las intuiciones del tiempo están supeditadas a la intuición espacial del desplazamiento. Así, Piaget concluye que "comprender el tiempo es liberarse del presente" (Piaget, 1992, p. 276), lo cual implica poder utilizar las regularidades identificadas en el pasado para anticipar el futuro y, asimismo, "desenvolver una sucesión de estados ninguno de los cuales es similar a los otros" (Piaget, 1992, p. 276). Por esa razón, la comprensión del tiempo requiere de la reversibilidad, en la medida en que es necesario que el sujeto trascienda el espacio para comprenderlo.

\section{En busca de la especificidad del pensamiento infantil: una crítica a la concepción piagetiana de la representación del tiempo en los niños}

La crítica que Merleau-Ponty realizará al análisis de Piaget en torno a la temporalidad infantil, se enmarca en la crítica que le realiza el fenomenólogo francés en torno a la representación del mundo en el niño. Sobre este punto, el enfoque que adoptará Merleau-Ponty se acerca a aquel adoptado por Henri Wallon (1949), quien se pregunta ante todo si los niños cristalizan su contacto con el mundo en algo parecido a una representación (Merleau-Ponty, 2001). Intentando encajar el pensamiento de los niños en categorías similares a las postuladas por Kant, Piaget concluye en una caracterización negativa del pensamiento infantil, el cual se ilustra y se valora en relación con los hitos del pensamiento adulto. Para Wallon, en cambio, el pensamiento infantil, no es un pensamiento categorial o de índole representativo, sino más bien un pensamiento polimorfo y confuso, aun no moldeado enteramente por la cultura. Para Piaget, con el desarrollo, se iniciaría una "marcha espontánea pero orientada de la representación del mundo físico" (Merleau-Ponty, 2001, p. 508). De esta forma, el desarrollo de la representación del mundo en el niño sería analizado por Piaget desde la óptica de un "físico adulto". Al respecto, Merleau-Ponty se pregunta: “ ¿No hay posibilidad de salir de la infancia sin llegar a ese estado del cual habla Piaget?"7 (Merleau-Ponty, 2001, p. 509).

Tomando la crítica que realiza Huang (1930) de la aproximación piagetiana al desarrollo infantil, Merleau-Ponty nota que Piaget no sólo incluye en su análisis a niños pertenecientes a un mismo medio social, sino que también los interroga sobre fenómenos de tal grandeza que ni siquiera los adultos podrían responder correctamente. De la misma manera, Piaget traduce la explicación que los niños dan sobre un fenómeno como su representación del mundo. Merleau-Ponty, en cambio, cuestiona este salto que Piaget da en la investigación misma poniendo en cuestión la noción de explicación suficiente. Para MerleauPonty, lo que define la representación en el niño (o, más bien, su caracterización del mundo) será la ausencia de una explicación suficiente; cuando el niño explicita un aspecto del mundo que lo rodea, no está dando una explicación en términos absolutos.

La crítica más fuerte que Merleau-Ponty desarrollará en torno a la concepción del tiempo desarrollada por Piaget, es aquella formulada por Wallon a partir de la descripción de lo que el psicólogo entiende como ultracosas. Tanto el tiempo como el espacio, están por fuera del alcance de la experiencia del niño. A estos objetos inabarcables por la experiencia misma, es aquello que Wallon denomina ultracosas:

Las ultracosas son los horizontes de realidad de los cuales el niño no duda, pero hacia los cuales no puede tener una actitud objetivante, como puede hacerlo con las cosas que están a su alcance. Esta idea es estructural; ella interesa a la configuración misma del universo infantil. (Merleau-Ponty, 2001, p. 512). 
Para Wallon, hay ultracosas e infracosas, las cuales son los horizontes internos de la experiencia, "un horizonte interior cercano de la estructura interna de las cosas visibles". Ambos horizontes están "en un estado de no condensación" y se caracterizan por ser difusos. Ese será, entonces, el carácter propio de la experiencia infantil, la cual estará abierta a este doble horizonte y no estará, tal como se creía desde la psicología genética, "llena de objetos". Por esa razón, Merleau-Ponty concluye que "Piaget parece cristalizar aquello que no es todavía sino contactos con los fenómenos que no han sido reducidos por ser observados" (Merleau-Ponty, 2001, p. 512). Algunos ejemplos de ultracosas son el cielo, las montañas, la tierra, el tiempo y el espacio. La advertencia que realiza Merleau-Ponty a partir de la caracterización de la experiencia infantil que realiza Wallon, es que en la medida en que la misma está atravesada por las ultracosas, no podremos referirnos a ella solamente con el lenguaje destinado a las cosas. Los niños nunca están insertos en el tiempo y el espacio objetivos (en un espacio-tiempo como cuadros vacíos). El tiempo es vivido como una "eternidad cíclica", como una preexistencia (Merleau-Ponty menciona el ejemplo en el que muchos niños consideran que las hojas de la primavera son hojas viejas que han regresado). Desde esta concepción del tiempo, es que podemos comprender la narración gráfica de los niños: los niños mezclan aquello que, en términos objetivos, sucedió de forma sucesiva. Para Wallon, entonces, no se trata de que los niños ignoran la estructura del tiempo objetivo, sino que poseen, en definitiva, "otra estructura del tiempo" (MerleauPonty, 2001, p. 512). Por lo cual, Merleau-Ponty concluye que la experiencia del niño está repleta de ultracosas, mientras que la de los adultos está constituida por cosas.

\section{Conclusión}

El dibujo infantil (así como la obra de algunos pintores impresionistas como Cézanne) revela para Merleau-Ponty el mundo vivido, el sujeto en situación. Las distintas perspectivas que confluyen en un mismo plano en la pintura impresionista dan cuenta de la ligazón entre movimiento y percepción y, de la misma forma, evocan cómo la situacionalidad del sujeto se hace presente en el acto mismo de conocer (Merleau-Ponty, 2001). Al mismo tiempo, el dibujo infantil da cuenta de las convenciones que atraviesan la representación espacial, revelando una nueva forma de comprender la objetividad: para Merleau-Ponty (1971), los dibujos de los niños son más "objetivos" que los de los adultos ya que hacen referencia a las circunstancias concretas que dieron origen a la representación, mientras que el dibujo de los adultos intenta reproducir el mundo "tal cual es", valiéndose de la perspectiva planimétrica como una herramienta unívoca e inequívoca de referencia al entorno. El mundo expresivo de los niños conmueve y pone en evidencia la premisa cartesiana que nos señalaba, con el ejemplo de la cera derritiéndose frente a nuestros ojos, que los sentidos no nos reportan un verdadero conocimiento de las cosas, condenando así durante años nuestra experiencia sobre el mundo. El dibujo del niño posee un lazo más estrecho con "lo real", con el mundo vivido, en tanto que da cuenta de un sujeto en situación. Es así como el acto expresivo de los niños nos devuelve una mirada naif sobre el mundo y nos resitúa como sujetos traspasados por una situación vital determinada: el naturalismo, en miras a brindar una imagen universal del mundo que nos rodea, ha terminado por convertirse en la más nociva de las ficciones, la cual nos ha sumido en el ocultamiento de nuestra experiencia y en la desvalorización de ese impulso mismo que nos lleva a la expresión.

Teniendo en cuenta estas consideraciones, el dibujo del niño adquiere desde la fenomenología otra significación. En primer lugar, debe ser considerado en sí mismo como un acto performático, esto es, como un flujo (jet) en donde lo más importante es la actividad misma implicada en su realización. El dibujo del niño, entonces, no posee ninguna intencionalidad explicativa, tal como podríamos identificar en los dibujos de un arquitecto. El dibujo "posee una realidad propia" y el niño encuentra placer en el hecho mismo de "significar por significar" (Merleau-Ponty, 2001, p. 513). Por lo tanto, en el dibujo, los niños se expresan, antes que expresar la cosa misma. El adulto considera que reconstruye su punto de vista sobre las cosas en un momento determinado; el niño, en cambio, "busca asir el núcleo único presente a todos los momentos de la historia" (Merleau-Ponty, 2001, p. 514) que está siendo narrada.

En segundo lugar, Merleau-Ponty toma de Luquet algunos puntos que aparecen hacia el final de su obra. En el dibujo, el niño nos enfrenta con una síntesis del objeto diferente a aquella que realiza 
el adulto: mientras que el adulto reconstruye un objeto incompleto (esto es, se limita a reproducir su propio punto de vista sobre el mismo), el niño intenta reunir en un mismo dibujo todos los aspectos que intervienen en la percepción misma de un objeto. De la misma manera que Cézanne lo hacía con sus pinturas, "el objetivo del dibujo infantil es entonces darnos la unidad de la cosa" (MerleauPonty, 2001, p. 516), intentando acercarnos a las distintas perspectivas implicadas en la percepción global de un objeto. En el dibujo, el niño presentifica en un mismo plano todas las perspectivas posibles del acto perceptivo, diluyendo entonces la relación figura-fondo que estará presente en todo objeto de la percepción. De la misma forma, esta concepción del dibujo infantil como acto mismo de expresión, confluye en una apreciación negativa del dibujo del adulto, el cual es considerado, inversamente, como un "empobrecimiento de lo percibido" (Merleau-Ponty, 2001, p. 517) ya que su creación es meramente una traducción punto por punto, de una "reducción analítica" de nuestro contacto con el mundo. En el dibujo en perspectiva realizado por el niño, no se intenta "sustituir" a la cosa misma, sino que allí se podrán encontrar al unísono todas las caras presentes en un objeto cuando este es percibido desde diferentes puntos de vista.

En tercer lugar, la experiencia del tiempo infantil descripta por Merleau-Ponty a partir del dibujo (y mencionada nuevamente en los cursos de la expresión, donde es traída a colación la descripción que Piaget hace de la vivencia del tiempo en el período sensorio-motor) es solidaria con la descripción del tiempo que presenta en la Fenomenología de la percepción (1984):

Si el observador, situado en una barca, sigue el hilo del agua, bien puede decirse que desciende con el curso del agua hacia su futuro, pero el futuro son los paisajes nuevos que le esperan en el estuario, y el curso del tiempo, no es ya la corriente misma: es el desenvolvimiento de los paisajes para el observador en movimiento. El tiempo no es, luego, un proceso real, una sucesión efectiva que yo me limitaría a registrar. Nace de mi relación con las cosas. (Merleau-Ponty, 1984, pp. 419-420).

En esta obra, la ficcionalidad del ordenamiento que le damos a nuestras vivencias en el mundo, es puesta de manifiesto. El mundo está constituido por una red de intencionalidades que "trazan el estilo de lo que va a venir". Mi campo perceptivo arrastra "su horizonte de retenciones y hace mella por sus pretensiones en el futuro". Con cada momento que viene, sostiene Merleau-Ponty, "el momento precedente sufre una modificación", se hunde "bajo la línea de los presentes" (MerleauPonty, 1984, p. 424). Por esa razón,

Las decisiones teóricas y prácticas de la vida
personal pueden captar a distancia mi pasado
y mi futuro, dar a mi pasado con todos sus
azares un sentido definido haciéndole seguir
un cierto futuro del que luego se dirá que era
la preparación, introducir la historicidad en
mi vida: este orden siempre tiene algo de
ficticio. (Merleau-Ponty, 1984, p. 358).

Introducir la historicidad en tanto orden causal en medio de lo que fue vivido de forma "azarosa", tendría algo del orden de lo creado, ya que el sentido que el pasado adquiere al ser rememorado difiere del sentido conferido en el momento en que es vivenciado. La ambigüedad temporal que nos atraviesa, hace de nuestra descripción del tiempo "un registro final", "el resultado de su paso", el cual intenta ser rescatado infructuosamente por el pensamiento objetivo. Solo tenemos meros registros de nuestra temporalidad, más no así una descripción plena de aquello que es el tiempo. Por ello, el paso del tiempo "es espacio", debido a que "sus momentos coexisten ante el pensamiento" y, el mismo tiempo, "es presente", ya que "la consciencia es contemporánea de todos los tiempos" (Merleau-Ponty, 1984, p. 423). Los niños, a diferencia de los adultos que ya se encuentran inmersos en las ilusiones estructurantes del objetivismo, lo expresan en su reconstrucción de la experiencia. Por ello, Merleau-Ponty sostiene:

\footnotetext{
El pasado no es, pues, pasado, ni el futuro, futuro. No existe más que cuando una subjetividad viene a romper la plenitud del ser en sí, y dibujar una perspectiva, introducir el no-ser en ella. Un pasado y un futuro surgen cuando me extiendo hacia ellos. (MerleauPonty, 1984, p.428).
}

El panta rei de Heráclito es interpretado por Merleau-Ponty como una de las "metáforas clásicas del tiempo", poniendo por sobre esta tesis su 
significación temporal antes que sus connotaciones ontológicas. Desde esta interpretación, el tiempo, como el río, forma una cosa consigo mismo. El tiempo se puede identificar como uno y el mismo en el curso de su devenir en la medida en que hay "una mirada" en su seno que le otorga unidad:

Hay un estilo temporal del mundo y el tiempo sigue siendo el mismo porque el pasado es un antiguo futuro y un presente reciente, el presente un pasado próximo y un futuro reciente, el futuro un presente $\mathrm{e}$ incluso un pasado por venir, eso es, porque cada dimensión del tiempo se trata o enfoca como algo diferente de ella misma; eso es, en fin, porque hay en la médula del tiempo una mirada. (Merleau-Ponty, 1984, p. 429).

El tiempo, entonces, "es alguien" en la medida en que las dimensiones que lo componen "expresan todas una explosión o un único impulso que es la mismísima subjetividad" (Merleau-Ponty, 1984, p. 430). Cuando Merleau-Ponty afirma que "hay que entender el tiempo como sujeto y el sujeto como tiempo" (Merleau-Ponty, 1984, p. 430), nos enfrenta no sólo con la imposibilidad de acceder a la temporalidad más allá de la vivencia que tengamos de ella, sino que hace de la expresión del sujeto en torno a su cauce, el testimonio más veraz de su flujo. De esta forma, el acto de expresión le es "esencial" al tiempo (al menos, claro está, para su comprensión) en tanto siempre se abre a un otro.

Asimismo, la temporalidad infantil es revalorizada como otra manera de vivir el tiempo, y no ya como una temporalidad adulta incipiente o meramente como un paso más hacia la objetivación del tiempo vivido. De esta forma, tiempo y espacio se presentan como dos dimensiones enlazadas en la experiencia infantil: para Piaget, en el estadio sensorio-motor, el tiempo es espacial; para Merleau-Ponty, la representación del espacio no puede abstraerse de la temporalidad. Por último, la concepción piagetiana de la temporalidad que refiere Merleau-Ponty en los cursos sobre la expresión como la forma de construcción del tiempo en la acción misma, no hace sino restringirse a la vivencia del tiempo que los niños experimentan durante el período sensorio-motor, en donde las velocidades no pueden ser comparadas entre sí y la distancia temporal solo puede ser referida como distancia espacial.

De esta forma, la experiencia estética infantil es presentada por Merleau-Ponty como eminentemente fenomenológica, como la representación del tiempo vivido que aún no ha sido asimilada por la culturalización y las formas estandarizadas de vivir el tiempo y el espacio 


\section{Referencias}

Álvarez Falcón, L. (2008). El régimen de la phantasia y la experiencia estética en Merleau-Ponty. Eikasía. Revista de Filosofía, IV(21), 83-107.

Battán Horenstein, A. (2010). La fenomenología de M. MerleauPonty y una crítica a la noción de representación. Representaciones. Revista de Estudios sobre Representaciones en Arte, Ciencia y Filosofía, 6(1). Recuperado de: http://www.academia.edu/1881504/ La_fenomenolog\%C3\%ADa_de_M._MerleauPonty_y_una_ cr\%C3\%ADtica_a_la_noci\%C3\%B3n_de_representaci\%C3\%B3n

Bimbenet, E. (2002). Les pensées barbares du premier âge. Merleau-Ponty et la psychologie de l'enfant. Chiasmi international, 4 (Merleau-Ponty. Figures et fonds de la chair), 65-86.

Carbone, M. (2011). Merleau-Ponty, la chair des images: entre peinture et cinéma. Paris, France: Vrin

Carretero, M. (1998). Introducción a la psicología cognitiva. Buenos Aires, Argentina: Aique.

Heidegger, M. (1996). El origen de la obra de arte. Madrid, España: Alianza.

Huang, I. (1930). Children's explanations of strange phenomena. Psychologische Forschung, 14, 63-182.

Merleau-Ponty, M. (1971). La prosa del mundo. Madrid, España: Taurus.

Merleau-Ponty, M. (1977). Sentido y sinsentido. Barcelona, España: Península.
Merleau-Ponty, M. (1984). Fenomenología de la percepción. Barcelona, España: Planeta-Agostini.

Merleau-Ponty, M. (2001). Psychologie et pédagogie de l'enfant. Cours de Sorbonne (1949-1952). París, France: Verdier.

Merleau-Ponty, M. (2002). El mundo de la percepción. Siete conferencias. Buenos Aires, Argentina: Fondo de Cultura Económica.

Merleau-Ponty, M. (2011). Le monde sensible et le monde de l'expression. Cours au Collège de France. Notes (1953). Genève, Suisse: MetisPresses.

Piaget, J. (1976). La toma de conciencia. Madrid, España: Morata. Piaget, J. (1978). Psicología del niño. Madrid, España: Morata. Piaget, J. (1992). El desarrollo de la noción de tiempo en el niño. D.F, México: Fondo de Cultura Económica.

Pinker, S. (2000). Cómo funciona la mente. Barcelona, España: Destino.

Saint Aubert, E. (2006). Vers une ontologie indirecte. Sources et enjeux critiques de l'appel à l'ontologie chez Merleau-Ponty. París, Francia: Vrin

Wallon, H. (1949). Les origines du charactère chez l'enfant. París, Francia: Quadrige / P.U.F.

Wallon, H. (1965). Fundamentos dialécticos de la psicología. Buenos Aires, Argentina: Proteo. 


\section{Notas}

1 Luquet distingue: el realismo fortuito, que refiere a un trazo realizado al azar y que posteriormente denominará "dibujo"; el realismo fallido, que corresponde al período en el que el niño advierte que puede representar o imitar objetos con sus dibujos pero aún no logra hacerlo debido a sus limitaciones motrices (el humo de la chimenea separado de la casa donde tiene origen, el "desmembramiento" de las partes de los animales, etc.); el realismo intelectual, donde el niño dibuja las representaciones intelectuales de las cosas, esto es, cómo cree que son y cuáles son sus elementos constitutivos, utilizando sobre todo la proyección (el muerto que se ve a través del ataúd, la comida en la panza de los animales, etc.); el realismo visual o estado terminal en el dibujo infantil, donde el niño comienza a articular los elementos de su producción y adquiere finalmente la perspectiva geométrica.

2 En español, suele traducirse también por "perspectiva militar".

3 En la actualidad, el egocentrismo identificado por Piaget en el periodo sensorio-motor es bastante discutido. Funciones tales como el mentir (acción que los niños realizan mucho antes incluso de la edad en la que debieran alcanzar la teoría de la mente) muestran que su pensamiento está mucho más centrado en los otros de lo que creemos. Al respecto ver: Carretero (1998, cap. 4).

4 La "toma de conciencia" es un concepto central dentro del campus teórico de Piaget, el cual hace referencia a "una construcción verdadera, que consiste en elaborar, no "la" conciencia considerada como un todo, sino sus diferentes niveles, como sistemas más o menos integrados" (Piaget, 1976, p. 9). No refiere, por ello, a una "incorporación" a un ámbito pre-dado.

5 Piaget cita dos experiencias diferentes: por un lado, los más pequeños eran invitados a ordenar figuras relativas al crecimiento de un árbol; por otro, los más grandes, debían poner en orden las imágenes correspondientes a una historia (imágenes de Dawid), las cuales eran ordenadas correctamente recién hacia los 8 años.

6 "Sobre 100 nuevos relatos, los siguientes porcentajes reproducen lo anterior: 5 años $90 \%, 6$ años $84 \%, 7$ años 30\%, 8 años 15\%, 9 años 11\%, 10 años 9\%" (Piaget, 1992, p. 265).

7 Este punto es desarrollado por Pinker en Cómo funciona la mente (2000, Cap. 5). Allí, Pinker cita una serie de experimentos ligados con nociones tales como la gravedad, que dan cuenta de la precariedad con la que los adultos lidian con ellas. Inversamente, experiencias realizadas con niños de menos de un año muestran cómo los bebés se manejan en el mundo a partir de nociones ligadas a la permanencia de la sustancia, la cual, para Piaget, aparece mucho más tarde en el desarrollo de las funciones cognitivas. 